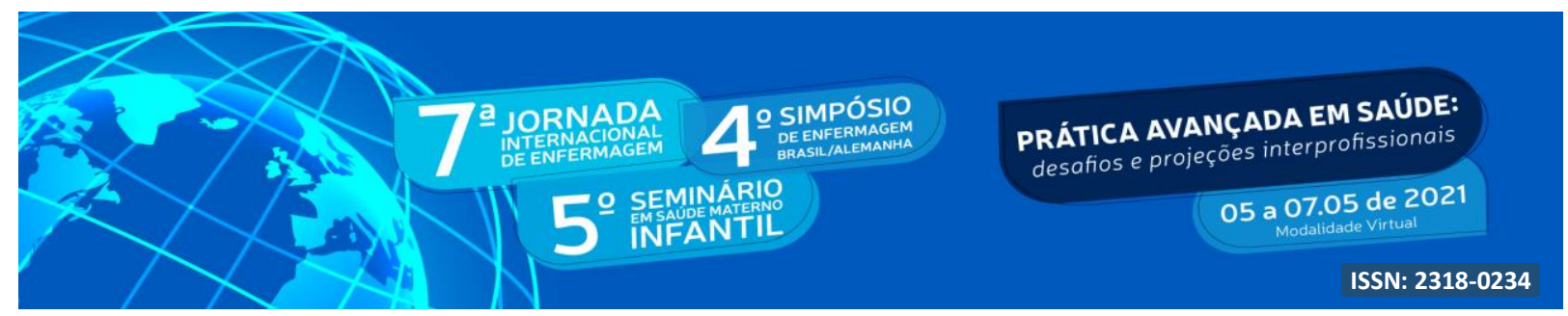

DOI: http://doi.org/10.48195/jie2021-166

\title{
A REGIONALIZAÇÃO DO PARTO E NASCIMENTO NA ATENÇÃO OBSTÉTRICA E NEONATAL ${ }^{1}$
}

\author{
Geiza Minello Marchezan²; Ana Sibila Dallabona³; Débora Diekel de Jesus Pessoa4; \\ Lígia Maria Terra Fontella ${ }^{5}$, Roseleia Regina Halmenschlager ${ }^{6}$; Claudia Maria Gabert \\ Diaz ${ }^{7}$.
}

\begin{abstract}
RESUMO
Objetivou discutir sobre a regionalização do parto e nascimento estabelecida pelos municípios da região central do Rio Grande do Sul (RS), na atenção obstétrica e neonatal. Trata-se de um estudo bibliográfico narrativo, desenvolvido em março de 2020, por meio de Manuais Ministeriais, Documentos Oficiais do RS e publicações científicas na Biblioteca Virtual em Saúde (BVS), com as palavras-chave: 'nascimento' and 'humanização da assistência' and 'politicas de regionalização'. O estudo dessa problemática integra o projeto de pesquisa Probic (UFN), intitulado "Boas práticas na atenção ao parto". A proposta de regionalização do parto e do nascimento visa qualificar a atenção à saúde da gestante e do recém-nascido, a fim de reduzir a mortalidade materno-infantil, além de resolver problemas relacionados a falta de estrutura em cidades de pequeno porte. Conclui-se que várias estratégias vem sendo desenvolvidas ao longo dos anos, entre eles a regionalização do nascimento, garantindo qualidade da atenção prestada.
\end{abstract}

Palavras-chave: nascimento; humanização da assistência; políticas de regionalização.

\begin{abstract}
It aimed to discuss the regionalization of childbirth and birth established by the municipalities of the central region of Rio Grande do Sul (RS), in obstetric and neonatal care. This is a narrative bibliographic study, developed in March 2020, through Ministerial Manuals, Official Documents of RS and scientific publications in the Virtual Health Library (VHL), with the keywords: 'birth' and 'humanization of assistance and regionalization policies. The study of this problem is part of the Probic research project (UFN), entitled "Good practices in childbirth care". The proposal for regionalization of childbirth and birth aims to qualify the health care of pregnant women and newborns, in order to reduce maternal and child mortality, in addition to solving problems related to the lack of structure in small cities. It is concluded that several strategies have been developed over the years, including the regionalization of birth, guaranteeing the quality of care provided.
\end{abstract}

Keywords: birth; humanization of assistance; regionalization policies.

\footnotetext{
${ }^{1}$ Artigo de Revisão Bibliográfica - Trabalho de Iniciação Científica - PROBIC/UFN.

${ }^{2}$ Estudante do Curso de Enfermagem - UFN. Bolsista PROBIC/UFN. geizaminellomarchezan@gmail.com

${ }^{3}$ Enf Obstetra, aluna do Mestrado Profissional em Saúde Materno Infantil - UFN. ana.sibila@ufn.edu.br

${ }^{4}$ Enf Obstetra, aluna do Mestrado Profissional em Saúde Materno Infantil - UFN.debestuda@ gmail.com

${ }^{5}$ Enf Obstetra, aluna do Mestrado Profissional em Saúde Materno Infantil - UFN. ligia.fontella@ufn.edu.br

${ }^{6}$ Ms. Enf Obstetra, egressa do Mestrado Profissional em Saúde Materno Infantil - UFN.

enfermeirarose@yahoo.com

${ }^{7}$ Dra. Enf Obstetra. Docente. Orientadora do Trabalho de Iniciação Científica - PROBIC/UFN.

cmgdiaz@ufn.edu.br 


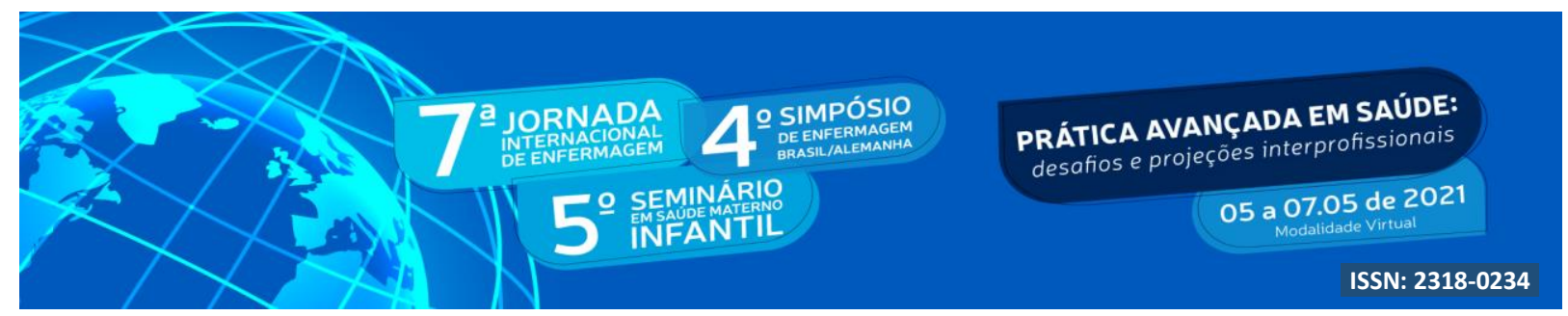

\section{INTRODUÇÃO}

O cuidado prestado à mulher durante o parto sofreu várias mudanças no decorrer dos tempos, resultando na institucionalização do parto devido aos avanços tecnológicos na área médica. No entanto, busca-se promover uma assistência humanizada às mulheres, considerando suas necessidades, bem como seus aspectos sócio-culturais, psicológicos e emocionais, que podem influenciar significativamente na vivência durante parto normal (LOPES; AGUIAR, 2020).

Mesmo com iniciativas ministeriais para a implementação de Políticas Públicas que busquem a melhoria da qualidade da assistência no ciclo gravídico puerperal, os índices de morbimortalidade materna e neonatal se constituem um desafio pelas suas elevadas taxas. A maioria dessas complicações e óbitos seriam evitadas se houvessem ações organizadas e integradas com cobertura abrangente, bem como utilização de tecnologias simples e economicamente viáveis (BRASIL, 2012).

Uma dessas iniciativas, constituem o processo de regionalização que contemplam uma lógistica de planejamento integrado, compreendendo os limites de territorialidade, identificando prioridades de intervenção e a disponibilidade dos sistemas funcionais de saúde, garantindo o acesso dos cidadãos a todas as ações e serviços necessários para a resolução de seus problemas de saúde, otimizando os recursos disponíveis (DUARTE et al., 2015). Nesta perspectiva, o Rio Grande do Sul (RS) conseguiu retomar a redução da mortalidade materna, muito possivelmente, em função da qualificação decorrente da regionalização da atenção ao parto e nascimento (WALCHER, 2017).

Esta temática tem estreita relação com a política de humanaização do parto e nascimento e com a Rede Cegonha, além de estar diretamente interligada com os objetivos do Milênio que une esforços para reduzir a Mortalidade Materna e Neonatal, o que justifica a relevância do estudo.

\section{OBJETIVO}

Discutir sobre a regionalização do parto e nascimento estabelecida pelos municípios da 


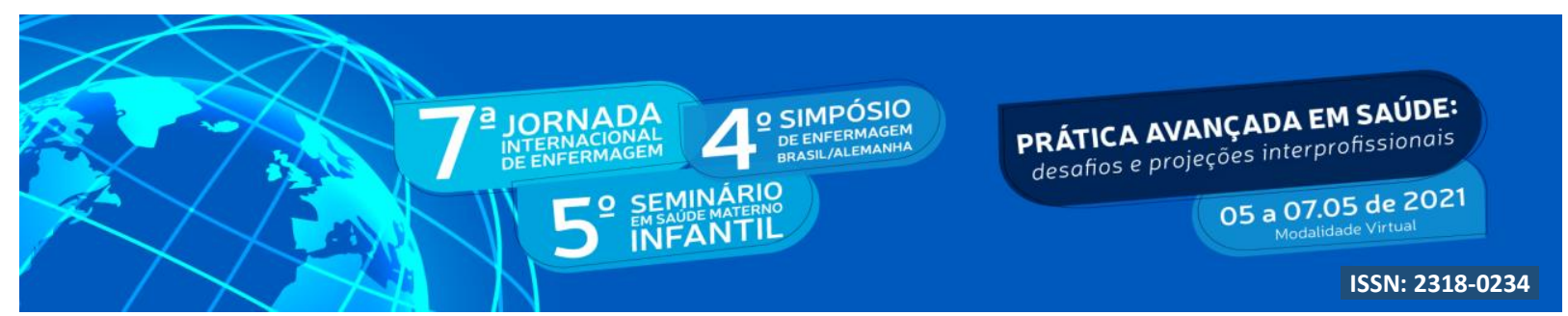

região central do Rio Grande do Sul (RS) na atenção obstétrica e neonatal.

\section{METODOLOGIA}

Estudo bibliográfico narrativo desenvolvido no em março de 2020, na Biblioteca Virtual em Saúde (BVS), com as palavras-chave: 'nascimento' and 'humanização da assistência' and 'politicas de regionalização'. Como critérios de inclusão: artigos completos, teses ou dissertações disponíveis na íntegra, gratuitos, no idioma português. Também foram utilizados Manuais Ministeriais e Documentos Oficiais do RS. Foram excluídos livros, publicações em inglês e espanhol.

Por meio de uma leitura criteriosa dos materiais bibliográficos encontrados, foi realizada a análise e reflexão acerca do tema, potencializando condições positivas e negativas a cerca da resolução da regionalização de saúde no contexto das maternidades. O estudo desse cenário integra o projeto de pesquisa Probic (UFN), intitulado "Boas práticas na atenção ao parto", a ser desenvolvido na região central do RS.

\section{RESULTADOS E DISCUSSÃO}

As diretrizes da atenção ao parto e nascimento no Brasil estão definidas pela Portaria GM/MS n 1459, que institui a Rede Cegonha, desde 2011, devendo ser implantada em todas as unidades da federação (BRASIL, 2011).

A política de regionalização de partos no Rio Grande do Sul, definida pela Resolução n 206/17 - CIB/RS instituída em 2017, propõe transferir partos de municípios de pequeno porte para centros de referência regionais, com a finalidade de qualificar a atenção à saúde da gestante, da assistência ao parto e ao recém-nascido, possibilitando a redução da mortalidade materna e infantil (SES/RS, 2017).

A região central do RS é composta por alguns municípios da $4^{\mathrm{a} C R S}$, da $8^{\mathrm{a}} \mathrm{CRS}$ e da $9^{\mathrm{a}} \mathrm{CRS}$. Na tabela 1, observa-se o posicionamento dessas coordenadorias e seus respectivos munícipios pertencentes: 


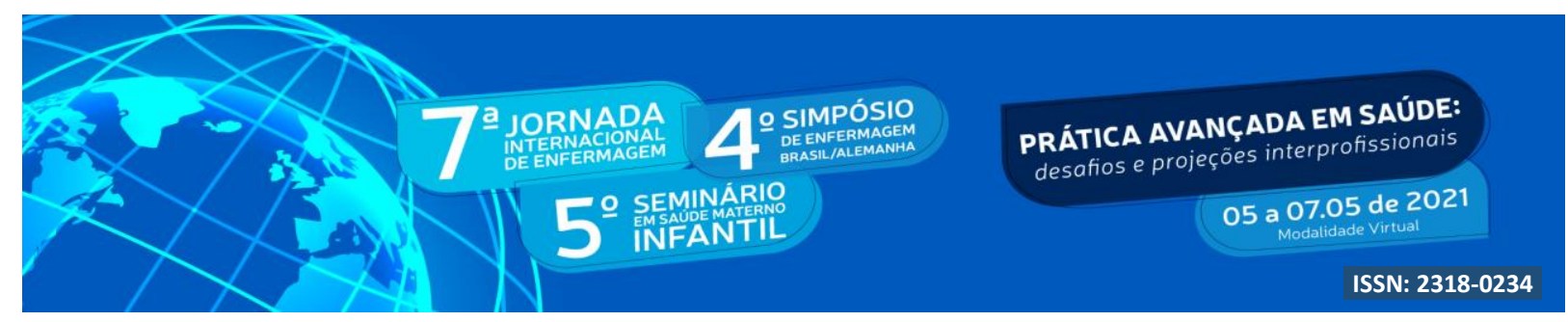

\begin{tabular}{|l|l|}
\hline $4^{\text {a }}$ CRS - Santa Maria & $\begin{array}{l}\text { Cacequi, Capão do Cipó, Dilermando de Aguiar, Dona Francisca, Faxinal do } \\
\text { Soturno, Formigueiro, Itaara, Ivorá, Jaguari, Jari, Júlio de Castilhos, Mata, } \\
\text { Nova Esperança do Sul, Nova Palma, Pinhal Grande, Quevedos, Restinga } \\
\text { Seca, Santa Maria, Santiago, São Francisco de Assis, São João do Polêsine, } \\
\text { São Martinho da Serra, São Pedro do Sul, São Sepé, São Vicente do Sul, } \\
\text { Silveira Martins, Toropi, Tupanciretã, Unistalda, Vila Nova do Sul (30 } \\
\text { municípios) }\end{array}$ \\
\hline $8^{\text {a CRS - Cachoeira do }}$ & $\begin{array}{l}\text { Agudo, Arroio do Tigre, Cachoeira do Sul, Cerro Branco, Encruzilhada do } \\
\text { Sul, Estrela Velha, Ibarama, Lagoa Bonita do Sul, Novo Cabrais, Paraíso do } \\
\text { Sul, Passa Sete, Segredo, Sobradinho (13 municípios) }\end{array}$ \\
\hline $9^{\text {Sa CRS - Cruz Alta }}$ & $\begin{array}{l}\text { Boa Vista do Cadeado, Boa Vista do Incra, Colorado, Cruz Alta, Fortaleza dos } \\
\text { Valos, Ibirubá, Jacuizinho, Quinze de Novembro, Saldanha Marinho, Salto do } \\
\text { Jacuí, Santa Bárbara do Sul, Selbach (12 municípios) }\end{array}$ \\
\hline
\end{tabular}

Fonte: SES/RS. Secretaria da Saúde do Estado do Rio Grande do Sul (BR). Plano diretor de regionalização da saúde. 2002.

A resolução supracitada tem como objetivo resolver problemas ligados a falta de estrutura em cidades de pequeno porte, as quais não possuem condições adequadas para parto e que, em muitos casos, não contam com médicos obstetras e pediatras plantonistas e com UTI Neonatal (SES/RS, 2017).

A proposta de regionalizar o parto para locais que possam realizar no mínimo 365 partos/ano vem sendo alicerçada a partir da análise de várias variáveis, que abrange diretrizes federais da Rede Cegonha, análise das condições exigidas pela vigilância em saúde, disponibilidade de profissionais que garantam a assistencia presencial, quantitativos mínimos de procedimentos sugeridos, viabilidade financeira do serviço, possibilidade de cofinanciamento estadual aos serviços de atenção ao parto e nascimento, acesso das gestantes a serviços localizados ao máximo $50 \mathrm{Km}$ de distância, com intuito de garantia do transporte a outras referências as situações de maior risco (WALCHER, 2017).

A política de regionalização de partos no RS ainda enfrenta resistência de municípios, de certa forma, necessitando de argumentos para incentivar a adesão, como a economia no aporte de recursos voltados à manutenção de equipes especializadas em locais que fazem poucos partos por ano.

\section{CONCLUSÃO}




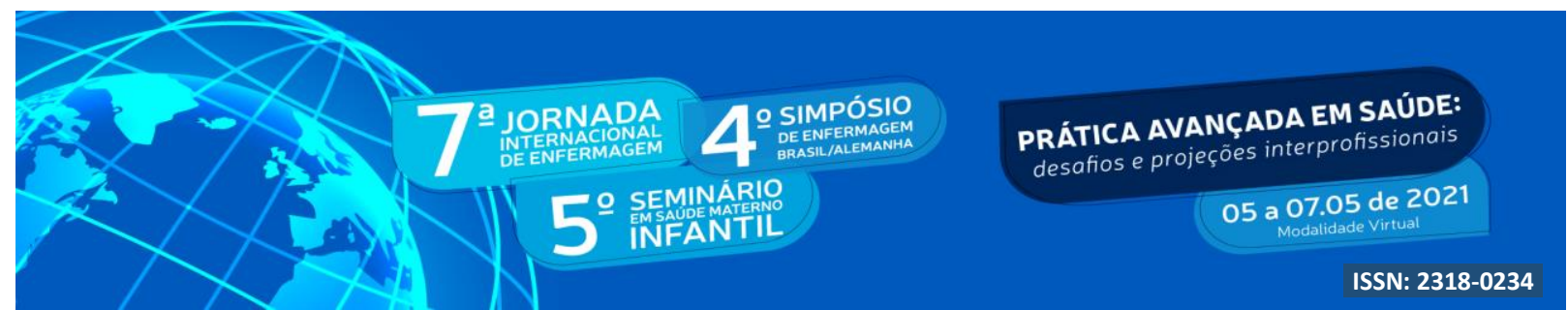

As importantes estratégias desenvolvidas ao longo desses anos, entre eles a regionalização do nasciemnto, não têm sido suficientes para garantir a qualidade da atenção prestada às mulheres, refletindo o efeito limitado das políticas públicas dirigidas à mudança do paradigma de assistência ao parto.

Com isto, os avanços na humanização da assistência ao parto, com a implementação das boas práticas deve estar amparada em políticas públicas de saúde e em evidências científicas, devendo ser praticada de forma efetiva por toda a equipe multiprofissional. Por fim, junto a este estudo, percebe-se a necessidade de que se tenham mais pesquisas acerca da assistência ao parto relacionadas as regionalizações da saúde ofertados nos dias de hoje, afim de confrontar e refletir resultados.

\section{REFERÊNCIAS}

BRASIL. Ministério da Saúde. Secretaria de Assistência à Saúde. Portaria No 1459 de 24 de junho de 2011. Institui, no âmbito do Sistema Único de Saúde - SUS - a Rede Cegonha. Saude Legis. 2011a. Brasília, 2011.

BRASIL. Ministério da Saúde. Secretaria de Atenção à Saúde. Departamento de Ações Programáticas Estratégicas. Gestação de alto risco: manual técnico / Ministério da Saúde, Secretaria de Atenção à Saúde, Departamento de Ações Programáticas Estratégicas. Brasília: Ministério da Saúde; 2012.

DUARTE, L. S. et al. Regionalização de saúde no Brsail: uma perspectiva de análise. Saúde Soc., v.24, n. 2, Apr-Jun 2015.

LOPES, L.C.S.; AGUIAR, R.S. Aplicabilidade das boas práticas de atenção ao parto: revisão integrativa de literatura. REVISA, v.9, n.1, p.: 133-43, 2020.

SES/RS. Secretaria da Saúde do Estado do Rio Grande do Sul (BR). Resolução no 206/17 -

CIB/RS. Pactua a organização da Rede de Atenção ao Parto e Nascimento de forma regionalizada, no Estado do Rio Grande do Sul. Porto Alegre; 2017. Disponível em: http://www.saude.rs.gov.br/upload/arquivos/201706/05110251cibr206-17.pdf. Acesso em: 27 mar. 2020.

SES/RS. Secretaria da Saúde do Estado do Rio Grande do Sul (BR). Plano diretor de regionalização da saúde; 2002. Disponível em:

https://www.mprs.mp.br/media/areas/gapp/arquivos/plano_diretor_regionalizacao_saude.pdf. 


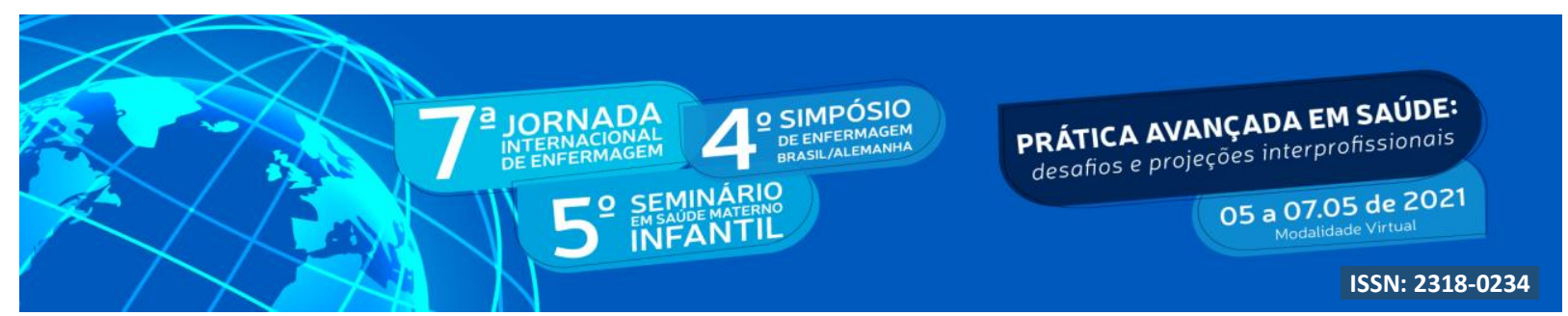

WALCHER, E. G. Impacto da Estratégia de Regionalização da Assistência ao Parto no Âmbito do Sistema Único de Saúde na Redução da Mortalidade Infantil no Estado do Rio Grande Do Sul. Dissertação (Mestrado). Universidade Federal do Rio Grande do Sul. Faculdade de Medicina. Programa de Pós Graduação em Saúde da Criança e do Adolescente. Porto Alegre, 2017. 